\title{
SOBRE LA PRESENCIA DE OXYPELTUS QUADRISPINOSUS BLANCHARD, 1851 (COLEOPTERA: CERAMBYCIDAE') EN FUEGO-PATAGONIA
}

\author{
ON THE PRESENCE OF OXYPELTUS QUADRIESPINOSUS BLANCHARD, \\ 1851 (COLEOPTERA: CERAMBYCIDAE) IN FUEGO- PATAGONIA
}

Alvaro Zúñiga-Reinoso² \& María Vanessa Lencinas ${ }^{3}$

Recientemente, Zúñiga-Reinoso (2013), realiza una revisión acabada de los cerambícidos de la región de Magallanes, donde menciona la necesidad de confirmar la presencia de algunas especies en esta región. Entre las especies mencionadas por dicho autor se encuentra Oxypeltus quadrispinosus Blanchard, 1851, cuya presencia en la región de Magallanes estaba dada por un registro antiguo en la literatura, sin localidad precisa (Peña, 1986) y por restos de élitros encontrados que fueron adscritos a O. quadrispinosus (Zúñiga-Reinoso, 2013). Ambos registros son indirectos y dudosos y con poca información sobre el hábitat al cual estaría asociada la especie. Por lo tanto, el objetivo de esta nota es registrar por primera vez a O. quadrispinosus en el sector boscoso de la isla Tierra del Fuego perteneciente a Argentina, describir su hábitat y discutir sobre su presencia en Fuego-Patagonia.

Material examinado: Argentina: Provincia de Tierra del Fuego, Antártida e Islas del Atlántico Sur: Ushuaia, Parque Nacional Tierra del Fuego. Georeferencia: 5451'35.67'S- 68³4'05.11'O. 2. 2. 2011. col. M.V. Lencinas. det. A. Zúñiga. Sobre Blechnum penna-marina (CADIC: 1 macho).
Descripción del hábitat: El ejemplar fue observado en un bosque mixto de Nothofagus betuloides- Nothofagus pumilio- Drymis winteri, que se encuentra al margen de un arroyo impactado por castores, en el sendero de la Baliza al que se accede desde Bahía Lapataia. La pendiente general de este sitio es de $5^{\circ}$, con exposición sur. Dicho bosque presentaba una densidad de 145 árboles/ha y 28 $\mathrm{m}^{2}$ de área basal, con una altura dominante de 18 m y un diámetro cuadrático medio de $48,1 \mathrm{~cm}$. La cobertura de copas era de 88,3\% y el índice de área foliar de 2,46, según datos relevados en esa fecha para ese manchón de bosque, encontrándose los árboles predominantemente en fase de desarrollo de envejecimiento. Adicionalmente, el $30 \%$ de la superficie del suelo en dicho sitio estaba cubierto de plantas vasculares, siendo las especies presentes: Acaena ovalifolia, Embotrium coccineum, Adenocaulon chilense, Maytenus magellanica, Osmorhiza depauperata, Ribes magellanicum, Taraxacum officinale, Viola magellanica, Dactylis glomerata, Trisetum spicatum, Codonorchis lessonii, Blechnum penna-marina, Maytenus disticha y regeneración de $N$. pumilio.

\footnotetext{
1 Por tradición y simplificación se seguirá la clasificación histórica del género en la familia.

${ }^{2}$ Programa de Doctorado en Ecología y Biología Evolutiva, Departamento de Ciencias Ecológicas, Facultad de Ciencias, Universidad de Chile. Santiago, Chile. alzure@gmail.com

3 Centro Austral de Investigaciones Científicas (CADIC), Houssay 200 (9410), Ushuaia. Tierra del Fuego, Argentina. mvlencinas@ gmail.com.
} 


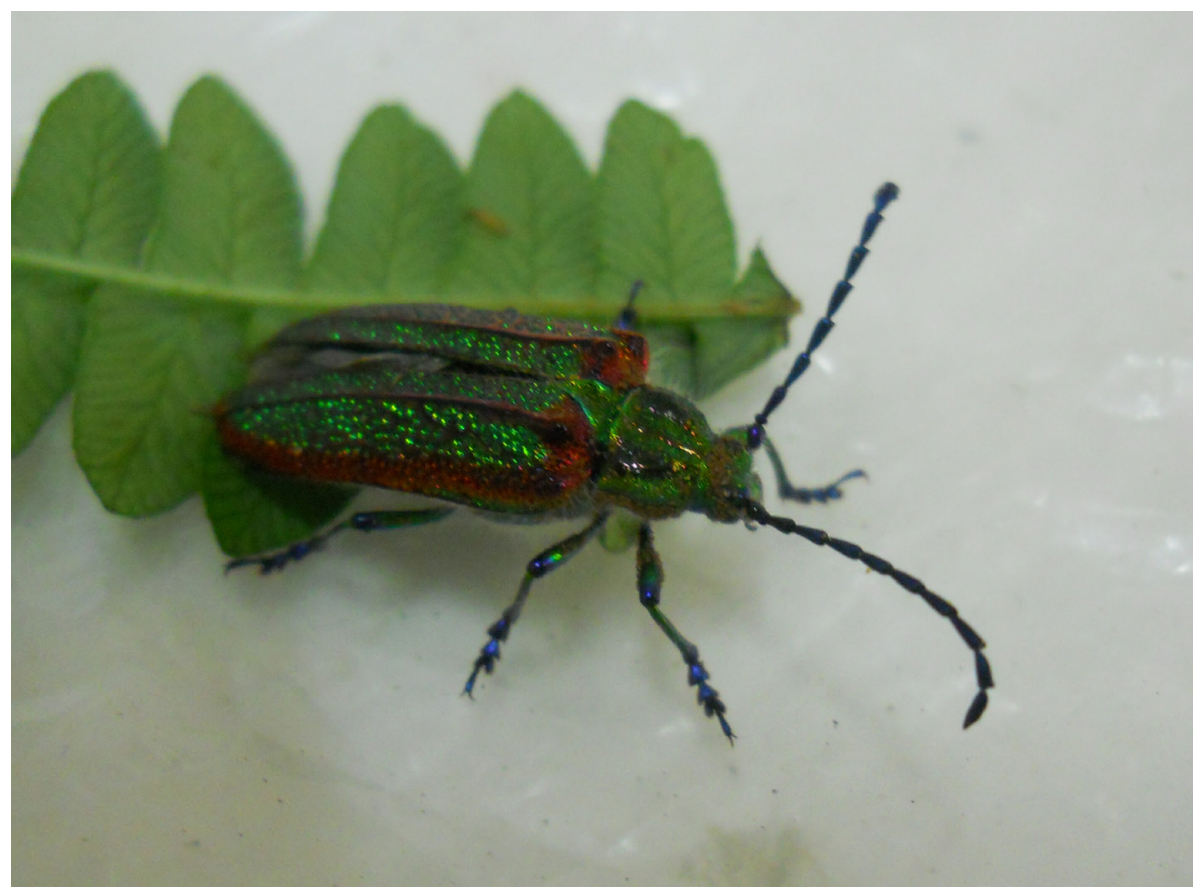

Fig. 1: Ejemplar macho de Oxypeltus quadrispinosus sobre la hoja de Blechnum penna-marina donde fue recolectado.

\section{COMENTARIOS}

Con este registro de un espécimen vivo se confirma la presencia de esta especie en las regiones australes de Fuego- Patagonia. Este registro se convierte en el más austral para Argentina y para la especies. El hábitat de O. quadrispinosus, sería de bosque subantártico de Nothofagus betuloidesNothofagus pumilio, el cual es un paisaje continuo que abarca todo el borde sur de Tierra del Fuego y de la región de Magallanes (Dimitri, 1972; Pisano, 1977; Dollenz, 1995; Roig, 1998) y el registro de Zúñiga-Reinoso (2013), en la porción norte de estos bosques, plantean que la presencia de esta especie sería en toda esta región austral de FuegoPatagonia. Se ha documentado que las larvas de $O$. quadrispinosus se desarrollan en la madera viva de Nothofagus dombeyi, N. pumilio y N. antárctica (Barriga et al. 1993), siendo las últimas dos especies pertenecientes a los bosques subantárticos. Por lo tanto, este coleóptero tiene estrecha relación con los bosques donde se desarrollan estas especies de árboles y su hallazgo en el suelo del bosque (i.e sobre B. penna-marina) sólo se trataría de un evento accidental.
Este registro hace entrever la necesidad de realizar mayores exploraciones entomológicas en la región, debido a que a pesar de que O. quadrispinosus es un insecto conspicuo en cuanto a tamaño y colorido, hasta la fecha no se tenía certeza de su presencia en la región. Por lo tanto, registros como este, son necesarios para complementar la información sobre la biodiversidad entomofaunística de las regiones australes.

\section{AGRADECIMIENTOS:}

El primer autor agradece a beca CONICYT N ${ }^{\circ}$ 21110367 para estudios de Doctorados Nacionales.

\section{LITERATURA CITADA}

Barriga, J., T. Curkovic, T. Fichet, J. Henríquez \& J. Magaya (1993). Nuevos antecedentes de Coleópteros xilófagos y plantas hospederas en Chile, con una recopilación de citas previas. Revista Chilena de Entomología, 20, 65-91. Bouchard, P., Y. Bousquet, A. E. Davies, M.A. Alonso-Zarazaga, J. F. Lawrence, C.H.C. 
Lyal, A. F. Newton, C.A.M. Reid, M. Schmitt, S. A. Adam Slipinski \& A.B.T Smith (2011). Family-group names in Coleoptera (Insecta). ZooKeys, 88, 1-972.

Cerda, M. (1986). Lista sistemática de los cerambícidos chilenos (Coleoptera: Cerambycidae). Revista Chilena de Entomología, 14, 29-39.

Dimitri, M. (1972). La Región de los Bosques Andino Patagónicos. Sinopsis General. INTA Buenos Aires, Argentina. 381 pp.

Dollenz, O. (1995). Los árboles y bosques de Magallanes. Punta Arenas, Chile: Ediciones de la Universidad de Magallanes.

Peña, L. (1976). Guía para reconocer los coleópte- ros de Chile continental. Expedición a Chile. Santiago, Chile: Editorial Nacional Gabriela Mistral.

Pisano, E. (1977). Fitogeografía de Fuego-Patagonia chilena. I. Comunidades vegetales entre las latitudes 51 y $56^{\circ}$ Sur. Anales Instituto $\mathrm{Pa}$ tagonia. 8: 121-250.

Roig, F. (1998). La Vegetación de la Patagonia. En: Correa, M. Flora Patagónica. INTA Colección Científica. Tomo VIII, vol I. Pp. 48-174.

Zúñiga-Reinoso A. (2013). Revisión de los Cerambycidae (Coleoptera: Cerambycidae) de la Región de Magallanes: Lista ilustrada. Anales del Instituto de la Patagonia. 40(1):64-70. 
Anales Instituto Patagonia (Chile), 2013. 40(1):22-27 